\title{
Origin of a novel protein-coding gene family with similar signal sequence in Schistosoma japonicum
}

\author{
Evaristus Chibunna Mbanefo ${ }^{1,6}$, Yu Chuanxin ${ }^{2}$, Mihoko Kikuchi ${ }^{1}$, Mohammed Nasir Shuaibu', Daniel Boamah',
} Masashi Kirinoki ${ }^{3}$, Naoko Hayashi ${ }^{3}$, Yuichi Chigusa ${ }^{3}$, Yoshio Osada ${ }^{4}$, Shinjiro Hamano ${ }^{5}$ and Kenji Hirayama ${ }^{* *}$

\begin{abstract}
Background: Evolution of novel protein-coding genes is the bedrock of adaptive evolution. Recently, we identified six protein-coding genes with similar signal sequence from Schistosoma japonicum egg stage mRNA using signal sequence trap (SST). To find the mechanism underlying the origination of these genes with similar core promoter regions and signal sequence, we adopted an integrated approach utilizing whole genome, transcriptome and proteome database BLAST queries, other bioinformatics tools, and molecular analyses.

Results: Our data, in combination with database analyses showed evidences of expression of these genes both at the mRNA and protein levels exclusively in all developmental stages of S. japonicum. The signal sequence motif was identified in 27 distinct S. japonicum UniGene entries with multiple mRNA transcripts, and in 34 genome contigs distributed within 18 scaffolds with evidence of genome-wide dispersion. No homolog of these genes or similar domain was found in deposited data from any other organism. We observed preponderance of flanking repetitive elements (REs), albeit partial copies, especially of the RTE-like and Perere class at either side of the duplication source locus. The role of REs as major mediators of DNA-level recombination leading to dispersive duplication is discussed with evidence from our analyses. We also identified a stepwise pathway towards functional selection in evolving genes by alternative splicing. Equally, the possible transcription models of some protein-coding representatives of the duplicons are presented with evidence of expression in vitro.
\end{abstract}

Conclusion: Our findings contribute to the accumulating evidence of the role of REs in the generation of evolutionary novelties in organisms' genomes.

Keywords: Signal sequence trap, Schistosoma japonicum, Repetitive elements, Gene duplication, Secreted proteins, Non-allelic homologous recombination

\section{Background}

Evolutionary novelties generated as an upshot of the "nascence" of new protein-coding genes are the bedrock of adaptive evolution and acquisition of novel molecular functions. The ever-growing vast and diverse protein repertoire in organisms can be ascribed to these events, and may explain the increasing heterogeneity among organisms of otherwise common ancestry [1-5]. Since the pioneering definitive treatise on gene duplication by Ohno about four decades ago [6], geneticists and evolutionary

\footnotetext{
* Correspondence: hiraken@nagasaki-u.ac.jp

'Department of Immunogenetics, Institute of Tropical Medicine (NEKKEN), and Global COE Program, Nagasaki University, 1-12-4 Sakamoto, 852-8523, Nagasaki, Japan

Full list of author information is available at the end of the article
}

biologists have advanced this traditional notion; creating remarkable insights into the composite patterns and underlying mechanisms of genetic innovations. Some of these mechanisms are illustrated in a supplementary figure (Additional file 1). The advent of the genomics era has most importantly armed scientists with a valuable tool to enhance discovery of the rather intriguing mechanisms underlying the "birth" of new genes [5].

Apart from the canonical gene duplication model as proposed by Ohno [6]; extensive studies in various organisms have not only elucidated other models of gene duplication, including "dispersed" duplication in addition to the more definitive "tandem" duplication [7-13]; but has also revealed multiple mechanisms leading to the emergence of new functional genes. These include but not limited to: recombination by exon shuffling or exon "scrambling" 
[4,14-18]; retrotransposition by retrotransposons yielding intronless chimeric genes [18-25]; transduction of genomic segments by transposable elements by skipping the characteristic weak polyadenylation signal in retrotransposons leading to the mobilization of adjacent genomic sequence; or may involve a repetitive element (RE) mediated DNA level recombination (DLR) by a nonallelic homologous recombination (NAHR) mechanism, in which the REs provide the requisite homologous sequences for the recombination of genomic sequences in a non-allelic manner [7,20,26-30]. Horizontal gene transfer between organisms although infrequent, can give rise to new genes in the recipient organism [31-33]. De novo origination of protein coding genes from previously non-coding genomic sequences is a very important mechanism previously underrated, but accumulating data in many organism show that this event occur more often than previously thought [2,3,34-40]. Equally, a new gene can arise from the fusion of two genes [1,3,22] or fission of a "parent" gene [41]. These mechanisms seldom operate singly as they frequently overlap, collaborating in the creation of nascent genes as depicted in the famous origins of Jingwei and Sphinx in Drosophila species [14,19].

Schistosoma japonicum along with S. mansoni and S. haematobium are the principal schistosome species causing human schistosomiasis. Uncharacteristic of other human invading schistosomes, S. japonicum is also able to infect several non-human mammalian hosts. While $S$. japonicum and S. mansoni inhabit the periportal veins and cause an intestinal form of the disease, characterized by liver granulomatous fibrosis as a consequence of host immune response to the eggs lodged in the hepatic sinusoids [42,43]; S. haematobium causes urinary schistosomiasis at the vesical bladder plexus. Although S. japonicum produces similar lesions like S. mansoni, the fibrotic lesions and hepatosplenomegaly, the most severe outcome of schistosomiasis, is relatively more frequent and severe in S. japonicum [44]. Also, in contrast to S. mansoni and S. haematobium, acute disease due to $S$. japonicum is common in endemic foci and is associated with severe and persistent manifestations that may rapidly progress the host mediated immunopathogenesis, terminating in a network of fibrotic lesions [45]. Secreted proteins from the parasite ova embolized in the liver of the host are accessible to the host immune cells being located at the host-parasite interface and thus constantly exposed to the host liver tissues. Such interactions play critical role in the initiation and progression of granuloma and fibrosis formation by mediating inflammation [42-45]. Secreted protein candidates thus, possess great potentials for application in interventions aimed at preventing severe hepatic pathogenesis [46,47] among other applications.
Nascent genes confer extra functional capacities for the organisms to confront the challenges of the ever dynamic environment, and may equally, albeit rarely, inflict some functional constraints. In any case, recently evolved characteristics could best be attributed to either: protein family or domains expansion, gene loss events [48], or more likely, evolution of new genes. S. japonicum relatively exhibit a higher degree of parasitism and dependence on host derived molecules and signals as inferred from genomic and transcriptomic studies [49-51]; it is able to infect a wide range of hosts, and produces relatively more severe pathogenesis [45]. While these could be attributed to a number of other factors including: selective pressure of parasite-host interactions, the extensive gene loss and protein domain elimination or expansion events observed in its genome and transcriptome [49]; the evolution of novel functional protein coding genes before and after the divergence from other members of the genus Schistosoma could account for these extra characteristics.

Here, we report putative evolutionary novel gene family of Asian schistosomes, S. japonicum on the premise that no homologs of the genes were found in the genome of its evolutionary close relatives in the genus Schistosoma, or in any other organism with a complete sequenced genome. The genes first caught our attention as genes bearing similar or same signal sequence from our previous work that identified some secreted protein coding genes from the eggs of S. japonicum using a signal sequence trap (SST) [47]. Given the available tools prior to the publication of the $S$. japonicum genome sequence, we had attributed this observation to some alternative or trans-splicing models. The present analysis was inspired by the availability of the invaluable tool presented by the recently published partially assembled genome of this parasite [49]. We adopted an integrated approach utilizing extensive BLAST queries and other bioinformatics tools, transcription and expression analyses, southern hybridization of genomic DNA and evolutionary analyses. We describe evidence of "genome-wide" dispersed duplication of a protein coding gene locus, which may have arisen recently from previously non-coding genomic sequence. The role of repetitive elements as major mediators of the dispersive duplication is analyzed and discussed. Detailed evidence of the potential transcription models of some protein-coding representatives of the duplicons with similar signal sequence is presented and supported by our observations. Finally, based on the identification of non-coding mRNA transcripts as alternatively spliced variants of protein coding mRNAs, we propose that the new genes could be under significant functional selection. 


\section{Results and discussion}

Sequence characteristics of a novel protein-coding gene family with similar signal sequences in S. japonicum

To identify secreted proteins from the eggs of S. japonicum, we previously utilized a signal sequence trap (SST) and isolated at least 15 full length $S$. japonicum egg stage cDNAs encoding secreted or membrane binding proteins [47]. In addition, we observed that six of these genes have same or similar signal sequences (Table 1) from our analyses in [47]. Multiple alignment of the initial SST isolated messenger RNAs (mRNAs) is presented as a supplementary information (Additional file 2), while the multiple alignment of the corresponding protein sequences showing the similar signal peptides is presented in Figure 1 with the phylogenetic tree of the SST identified family members. Given the available tools at the time we made this observation, we had attributed this trend to some alternative splicing or trans-splicing models. Here, we took advantage of the recently characterized and published partial assembly of the genome sequence and transcriptome of $S$. japonicum to unravel the possible underlying mechanisms of signal sequence similarity among SST identified genes. BLASTN search on the whole non-redundant (nr) nucleotide collections and all expressed sequence tags (ESTs) in GenBank including the $S$. japonicum transcriptome using the similar signal sequence as query showed that a total of 181 mRNA sequences and 14 ESTs all belonging to S. japonicum bear the similar signal sequence. Based on information in the UniGene database that provides sets of transcript sequences that appear to come from the same transcription locus, these mRNA sequences with similar signal sequence were placed in 27 distinct UniGene entries (Table 2). By further sequence alignments of the returned mRNA sequences and information from UniGene, we grouped the mRNA transcripts according to their gene products and identified at least 7 distinct egg proteins, somula protein, 53 other hypothetical protein sequences and 10 non-coding mRNAs, all bearing the similar signal motif. All protein products of the mRNAs in the public database bearing the similar signal sequence were characteristically short, with one of them containing only 54 amino acids residues. A genome wide BLAST search using the similar signal sequence as query against all whole genome shotgun (WGS) reads also produced hits on 34S. japonicum genome contigs (Table 3) distributed within 18 genome scaffolds (Table 4), thus confirming the existence of such sequences in the genome at multiple loci. These loci were non-redundant and non-overlapping as confirmed from the partially mapped scaffolds of this parasite's genome accessible in GeneDB [52]. For clarity, we restricted further analyses to the initial cDNAs we had identified from our previous study using the SST.

To assess whether some homologs or at least some similar domains exist in other species, BLASTN and BLASTP searches using both the signal sequence and the entire coding sequences of the mRNAs and protein sequences as queries showed that these genes have no homologs in any other organism, but their expression in S. japonicum is supported by evidence from transcriptome and proteomic data. A search on several protein domain databases showed that although our candidates were classified in the same protein family with similar domains and assigned to a domain ID (ProDom:PD884968), no related domain or protein family was found in any other organism. The absence of these genes in the genome of S. mansoni, S. haematobium and other published genomes cannot possibly be attributed to sequencing gaps or annotation errors since the WGS sequencing approach is considerably reliable [49], and the fact that we adopted a multiple species approach covering the entire available sequenced genomes of all species makes this even more improbable $[37,40]$. Given the accumulating evidences of de novo origin of new genes from previously non-coding DNA sequences [2,34-40], we propose that the coding sequence of these genes may have recently originated de novo from previously non-coding DNA sequences in the ancestral forms, and subsequently duplicated and dispersed in the genome. This represents a more plausible interpretation than the improbable alternative hypothesis of concurrent gene deletion or inactivation in multiple ancestral lineages.

Table 1 *SST isolated S. japonicum egg CDNAs with similar signal peptide

\begin{tabular}{llll}
\hline Gene Products & GenBank cDNA Accession & GenBank Protein Accession & Signal Peptide \\
\hline SjCP1084 & AY570737 (1027 bp) & AAS68242 (271aa) & MRIINLVIISTALLLINLLQTKSQ \\
SjCP3611 & AY570744 (983 bp) & AAS68249 (260aa) & MRIIILGIISTVLLLINLLQTKSQ \\
SjCP501 & AY570753 (1038 bp) & AAS68258 (174aa) & MRIINLVNISTVLLLINLLQTKSQ \\
SjCP3842 & AY570748 (854 bp) & AAS68253 (203aa) & MFKMRIINLVNISTVLLLINLLQTKSR \\
SjCP400 & AY570756 (848 bp) & AAS68261 (124aa) & MFKMRIINLVNISTVLLLINLLQTKSQ \\
SjCP1531 & AY570742 (1037 bp) & AAS68247 (274aa) & MFKVRIINLVNISTVLLLINLLQTKSQ \\
\hline
\end{tabular}

*SST: Signal Sequence Trap. 


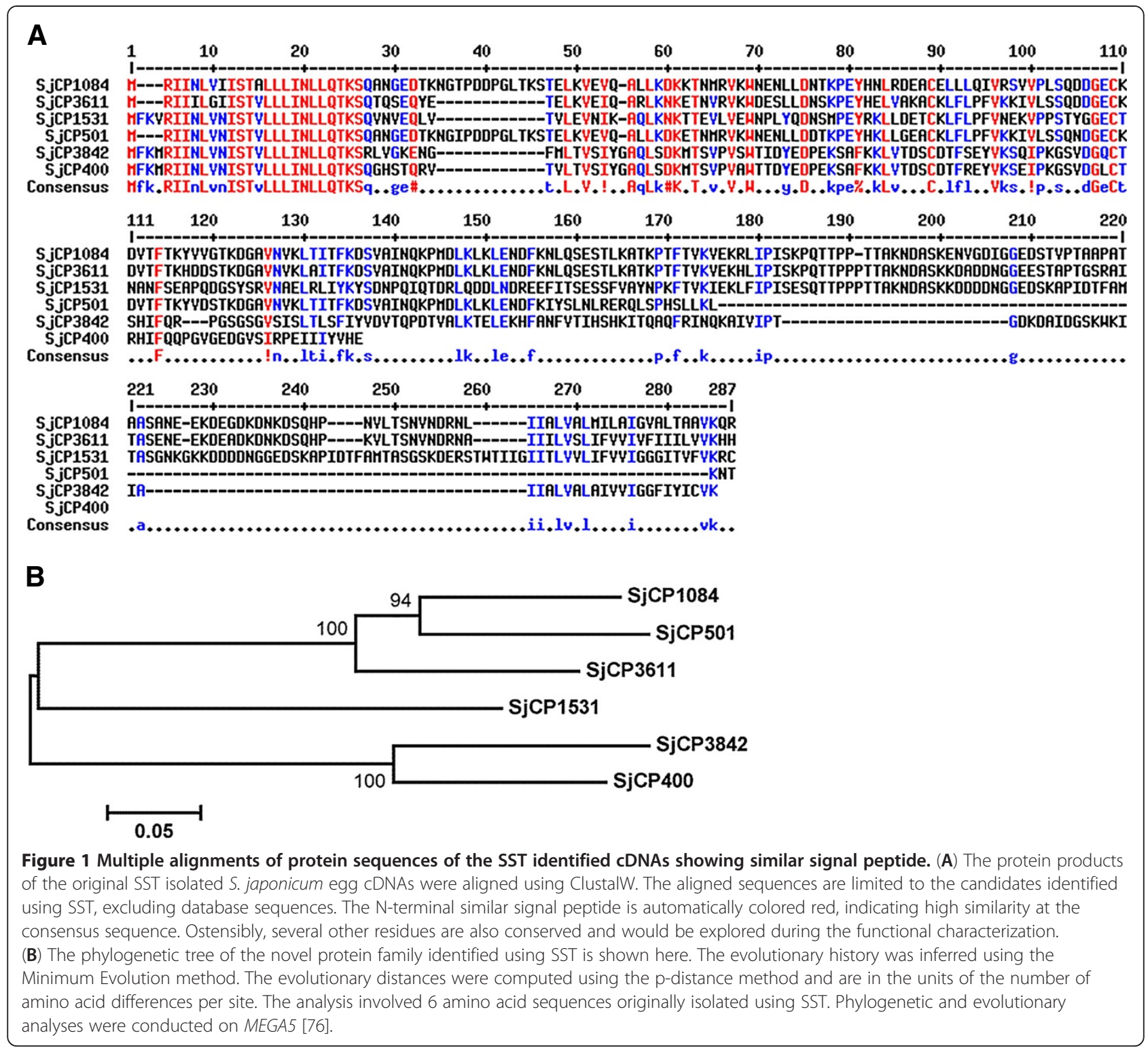

\section{Species and strain specific expression}

To further exclude the possibility of false negative observations, we assessed the presence of the gene loci among different species and strains of Schistosoma in vitro using southern blots. This genomic locus and its duplicons was found to be exclusively present in all the strains of $S$. japonicum using southern hybridization experiment utilizing genomic DNA samples of different strains of S. japonicum (Japanese, Chinese and Philippines), and other species of Schistosoma including S. mansoni, S. haematobium and S. mekongi (Figure 2), covering all the major clades in the genus. The result of southern hybridization using 462 base-pair digoxigenin labeled hybridization probe containing the similar signal sequence and designed to be specific to the gene loci under consideration showed that this genomic sequence was not found in any other species of Schistosoma except in all strains of S. japonicum analyzed. Several bands representing the duplicated loci are apparent in the hybridized blots (Figure 2). The analyzed samples is composed of representatives of the species complexes of this genus and further provide insight into the inter-species, intraspecies and intra-strain variations that may exist among the members of the genus Schistosoma. In line with the widely accepted Asian origin hypothesis deduced from the evolutionary biogeography of this genus as inferred from evidences at the morphological, karyotype and molecular levels [53], it is highly plausible that this genomic sequence has recently evolved exclusively within the S. japonicum complex long after the divergence of the ancestors of the African species and other re-invading Asian species with origin from Africa [53,54]. The fact 
that this gene locus was completely lacking in other Asian species like S. mekongi of common ancestry with $S$. japonicum even throws more light on the most probable evolution of these other Asian species which are thought to have evolved from same ancestor or as descendants of S. japonicum based on mitochondrial gene arrangement [55]. Either S. japonicum and other Asian species in the $S$. japonicum group evolved independently

Table 2 *UniGene entries for S. japonicum mRNAs and ESTs bearing the similar signal sequence $(n=195)$

\begin{tabular}{|c|c|c|c|}
\hline $\begin{array}{l}\text { UniGene } \\
\text { Name }\end{array}$ & $\begin{array}{l}\text { UniGene ID } \\
\text { (UID) }\end{array}$ & Set of likely mRNA transcripts (GenBank) & $\begin{array}{l}\text { Gene Products } \\
\text { (Database annotation) }\end{array}$ \\
\hline Sja.1526 & 1476162 & AY814448, BU780442 $2^{\text {est }}$ & Egg protein SjCP3611 \\
\hline Sja.1611 & 1476247 & FN317637, BU772954 ${ }^{\text {est }}$ & Hypothetical protein \\
\hline Sja.1628 & 1476264 & AY570742, FN320556, FN320555, FN320553, FN320552, FN320551, FN320550, FN320549 & Egg protein SjCP1531 \\
\hline Sja.1676 & 1476312 & $\begin{array}{l}\text { AY570748 SST , AY223245, AY222916, AY813542, EF127834, EF140742, FN323799, FN323800, FN323801, } \\
\text { FN323803, FN323793, FN323792, FN323791, FN323790, FN323788, FN323785, FN323782, FN323781, } \\
\text { FN323779, FN323778, FN323777, FN323776, FN323773, FN323772, FN323771, FN323770, FN323769, } \\
\text { FN323768, FN323767, FN323766, FN323765, FN323764, FN323763, FN323762, BU772060 } \\
\text { BU766145 , }\end{array}$ & Egg protein SjCP3842 \\
\hline Sja.2063 & 1476798 & FN321064, FN321061 & Egg protein SjCP1084 \\
\hline Sja.2065 & 1476800 & $\begin{array}{l}\text { AY570753 }{ }^{\text {SST }}, \text { AY } 570744^{\text {SST }}, \text { AY814685, FN327232, FN327137, FN318042, FN321065, FN321060, } \\
\text { FN321059, FN321058, FN321057, FN321056, FN321055, FN329815 }{ }^{\text {nc }}, \text { BU768978 }\end{array}$ & $\begin{array}{l}\text { Egg protein SjCP3611, } \\
\text { Egg protein SjCP501, } \\
\text { Hypothetical proteins }\end{array}$ \\
\hline Sja.2070 & 1476805 & AY599749 SST & Egg protein SjCP1731 \\
\hline Sja.5326 & 2034920 & FN326953, FN330298 nc & Hypothetical protein \\
\hline Sja.9771 & 2493712 & $\begin{array}{l}\text { AY570756 SST, FN327121, FN327254, FN327253, FN327241, FN327233, FN327229, FN327224, } \\
\text { FN327222, FN327216, FN327196, FN327185, FN327163, FN327158, FN327154, FN327129, } \\
\text { FN327125, FN327115, FN327089, FN327083, FN327073, FN327057, FN327050, FN327049, } \\
\text { FN327045, FN327042, FN327035, FN327022, FN327018, FN327014, FN327000, FN326998, } \\
\text { FN326978, FN326973, FN326961, FN326960, FN326959, FN326930, FN326905, FN326883, } \\
\text { FN326882, FN326881, FN326859, FN326857, FN326852, FN326851, FN326841, FN326831, } \\
\text { FN326829, FN326822, FN326808, FN326801, FN326790, FN326770, FN326740, FN330540 }\end{array}$ & $\begin{array}{l}\text { Egg protein SjCP400, } \\
\text { Somula protein }\end{array}$ \\
\hline Sja.11083 & 2671933 & $\begin{array}{l}\text { AY915467, FN327219, FN327063, FN326828, FN326826, FN323794, FN323797, FN323798, } \\
\text { FN323802, FN323789, FN323787, FN323786, FN323784, FN323783, FN323780, FN323774, } \\
\text { FN323761, FN323760, FN323759, FN323758, FN323757, FN320521, FN320520, FN320519, } \\
\text { FN320518, FN320517, FN320516, FN320515, FN320513 }\end{array}$ & $\begin{array}{l}\text { Egg protein SjCP3842, } \\
\text { Hypothetical protein }\end{array}$ \\
\hline Sja.11325 & 2672175 & $\begin{array}{l}\text { AY813755, FN320057, FN320056, FN320514, FN329566 }{ }^{\text {nc }}, \text { BU768160 }^{\text {est }}, \text { BU774105 }^{\text {est }}, \text { BU770186 }^{\text {est }} \\
\text { BU779051 }\end{array}$ & $\begin{array}{l}\text { Egg protein SjCP3842, } \\
\text { Hypothetical protein }\end{array}$ \\
\hline Sja.11840 & 2895838 & FN327242, FN327131, FN327087, FN326854, BU776301 $1^{\text {est }}$ & Hypothetical protein \\
\hline Sja.11891 & 2895889 & AY813975, FN329814 ${ }^{\text {nc }}$, BU769048 $8^{\text {est }}$ & Egg protein SjCP1084 \\
\hline Sja.13298 & 3987026 & FN320059 & Hypothetical protein \\
\hline Sja.13324 & 3987052 & AY570737 ${ }^{\text {SST }}$, FN328299 ${ }^{\text {nc }}$ & Egg protein SjCP1084 \\
\hline Sja.13882 & 3987610 & FN330716 ${ }^{\text {nc }}$ & None \\
\hline Sja.13956 & 3987684 & FN330422 $2^{\text {nc }}$ & None \\
\hline Sja.14071 & 3987799 & FN329677 & None \\
\hline Sja.14095 & 3987823 & FN329269 ${ }^{\text {nc }}$ & None \\
\hline Sja.14561 & 3988289 & FN327139, FN323795, FN323796, FN323775 & Egg protein SjCP3842 \\
\hline Sja.14562 & 3988290 & FN327130, FN326955, FN326901 & Egg protein SjCP1084 \\
\hline Sja.14565 & 3988293 & FN327099 & Egg protein SjCP1084 \\
\hline Sja.14614 & 3988342 & FN320058 & Hypothetical protein \\
\hline Sja.14627 & 3988355 & FN319007 & Hypothetical protein \\
\hline Sja.14941 & 3988669 & FN320554 & Hypothetical protein \\
\hline Sja.15036 & 5233761 & FN326786, FN318043, CX861530 est & Hypothetical protein \\
\hline Sja.15108 & 5233833 & AY810465, FN321062 & Hypothetical protein \\
\hline
\end{tabular}

* UniGene is a database of sets of transcript sequences that appear to come from the same transcription locus. The original set of CDNAs we earlier identified using signal sequence trap bear the superscript tag ${ }^{\text {SST }}$ ).

Transcripts with tags $\left({ }^{\text {est }}\right)$ and $\left({ }^{\text {(c) }}\right)$ are expressed sequence tags (ESTs) and non-coding mRNAs respectively. 
from a common ancestor, or the evolution of this locus and the subsequent dispersed duplication occurred recently after the other Asian forms have diverged (see phylogenetic relationship in Additional file 3). An alternative explanation is that the gene was not fixed or was deleted from the genome of the other Asian and African species. Since the last hypothesis is highly unlikely, we concluded that our observation was a product of a newly evolved gene locus possibly from mutations or modifications on a previously non-coding sequence in the ancestral forms, which was subsequently severally amplified and dispersed in the genome of S. japonicum after all other species of the genus had diverged. Furthermore, a close look at the banding pattern of the restriction digested genomic DNA of different strains of S. japonicum as observed in the southern blotting result revealed that possible intra-species and intra-strain genetic variations could exist among the members of the species complex (Figure 2). Whether the S. japonicum complex (Japanese, Chinese, Philippines and animal infecting Formosa strains) is made up of four geographical strains, four subspecies or four independent biological species remains contentious. Be that as it may, this presents an interesting subject for further research and could be further explored using a wider array of isolates from different regions.

Nevertheless, while it is completely normal to verify this exclusive evolution and dispersed duplication hypothesis by confirming the physical localization of the gene loci in the genome and chromosomes by performing synteny analysis, we are unable to achieve this because we do not have access to a fully mapped chromosome information of the genome of S. japonicum. However, the distribution of the contigs and scaffolds bearing the similar signal sequence apparently suggests a dispersed distribution. To confirm this hypothesis and to exclude the possibility of overlapping among the loci, we generated the restriction map of six of the genome scaffolds bearing duplicated loci based on the information on the genome map, performed southern hybridization using restriction endonuclease digested genomic DNA from S. japonicum species and strains; and were able to match the expected probe binding fragment sizes with the observed bands on the hybridization blots (Additional file 4). Also an ancestral homolog is required for synteny analysis, however, we could not find a homolog in S. mansoni, another member of the genus with sequenced genome; and the genome of other more closely related species like $S$. mekongi and S. malayensis are not yet sequenced. Unless new evidences emerge from future updates in the sequenced genomes, we hold true that these genes have newly evolved, probably from modifications on previously non-coding ancestral DNA sequences and subsequently disperse duplicated. As opined in previous studies, the short length of our identified genes is an expected property of nascent genes because of improbability of evolution of long open reading frames (ORFs) and the complexity of intron splicing signal [38]. We expect these novel genes to be of functional significance since new genes tend to display accelerated sequence and structural changes towards neo-functionalization [1], and most newly characterized genes from other species have been shown to be characteristically functional $[35,56]$. Other workers showed that the common pathway for de novo protein-coding gene evolution involves a piece of DNA sequence to be transcribed via recruitment of all transcription core promoters, other elements and machines; followed by the acquisition of a translatable ORF through mutations or other sequence alteration mechanisms $[2,35]$. Together, our findings support the presence of these intrinsic features of novel genes in the identified candidates, including the gradual model of novel protein coding gene origination.

\section{Evidence of dispersed duplication from a source gene locus}

The mechanisms behind dispersed duplication could be hidden within the DNA sequences of the duplicates or the adjacent flanking genomic sequences. In line with this, we explored the DNA sequences of the gene loci and the surrounding genomic sequences to identify possible mechanisms underlying dispersed duplication proposed in our hypothesis. A genome-wide BLAST search against WGS reads using the similar signal sequence as query returned 34 contigs of varying lengths and degrees of degradation (Table 3). By manually tracing these 34 contigs to the genome scaffolds, we found that they were distributed within 18 scaffolds (Table 4), apparently widely dispersed in the genome of S. japonicum as inferred from the genome map. To explore the mechanism of such dispersed duplication of a genomic sequence, a comparative analysis involving a parent gene in an ancestral species is often required. However, since we were unable to find any parental homolog in the available genome data and proteomes, and because gene duplication produces a diverse set of progeny loci with varied degrees of homology to an ancestral source locus when it exists [9], we performed a comparative sequence analysis on the 34 contigs as representatives of the gene loci. The result revealed a particular prominent contig in the $S$. japonicum WGS reads [GenBank: CABF01020060], the longest of the set of "duplicons" (43.7 kb), which significantly encompassed the length of the other contigs (Figure 3). CABF01020060 was therefore putatively selected as the duplication 'source locus' and utilized as such for most of the analyses performed in this study. 
Table 3 Schistosoma japonicum genome contigs containing the similar signal sequence $(\mathbf{n}=34)$

\begin{tabular}{|c|c|c|c|}
\hline${ }^{*}$ Contig [GenBank Accession No.] & $\begin{array}{l}\text { Transcription } \\
\text { Strand }\end{array}$ & Size, kb & $\begin{array}{l}\text { Signal Sequence } \\
\text { coordinates }\end{array}$ \\
\hline SJC_C002611 [CABF01002612] & - & 69.7 & $4848-4779$ \\
\hline SJC_C002621 [CABF01002622] & - & 0.6 & $276-205$ \\
\hline SJC_C002622 [CABF01002623] & - & 1.6 & $999-928$ \\
\hline SJC_C002627 [CABF01002628] & - & 3.0 & $2413-2342$ \\
\hline SJC_C002629 [CABF01002630] & - & 14.5 & $3856-3785$ \\
\hline SJC_C013669 [CABF01013761] & - & 6.8 & $3284-3217$ \\
\hline SJC_C019814 [CABF01020047] & + & 29.2 & $19023-19094$ \\
\hline SJC_C019817 [CABF01020050] & - & 10.1 & $6502-6431$ \\
\hline SJC_C019827 [CABF01020060] & - & 43.7 & $42511-42440$ \\
\hline SJC_C022876 [CABF01022876] & - & 12.4 & $9335-9264$ \\
\hline SJC_C022884 [CABF01022884] & + & 12.9 & $493-564$ \\
\hline SJC_C023364 [CABF01023364] & - & 12.4 & $10498-10427$ \\
\hline SJC_C025268 [CABF01025296] & - & 12.1 & $7860-7789$ \\
\hline SJC_C027826 [CABF01027854] & + & 4.3 & $433-504$ \\
\hline SJC_C027833 [CABF01027861] & - & 11.9 & $5768-5697$ \\
\hline SJC_C027838 [CABF01027866] & + & 19.0 & $12367-12438$ \\
\hline SJC_C032855 [CABF01032892] & - & 22.3 & $383-322$ \\
\hline SJC_C032859 [CABF01032896] & - & 9.6 & $4663-4602$ \\
\hline SJC_C043165 [CABF01043187] & + & 4.9 & $2484-2544$ \\
\hline SJC_C057153 [CABF01057161] & + & 2.8 & $337-408$ \\
\hline SJC_C061392 [CABF01061395] & - & 7.4 & $4411-4342$ \\
\hline SJC_C067189 [CABF01067176] & + & 4.9 & $388-459$ \\
\hline SJC_C067567 [CABF01067411] & - & 3.2 & $925-854$ \\
\hline SJC_C070280 [CABF01070230] & - & 6.8 & $271-200$ \\
\hline SJC_C072631 [CABF01072590] & + & 1.9 & $1646-1717$ \\
\hline SJC_C072632 [CABF01072591] & + & 2.6 & $446-517$ \\
\hline SJC_C073741 [CABF01073691] & - & 2.4 & $2389-2319$ \\
\hline SJC_C075160 [CABF01075030] & - & 6.7 & $5231-5160$ \\
\hline SJC_C076469 [CABF01076032] & + & 3.1 & $1295-1366$ \\
\hline SJC_C077101 [CABF01078976] & - & 1.1 & $656-585$ \\
\hline SJC_C080985 [CABF01080674] & + & 2.3 & $1094-1165$ \\
\hline SJC_C081391 [CABF01080757] & - & 2.1 & $1918-1847$ \\
\hline SJC_C081246 [CABF01080893] & - & 1.3 & $1131-1060$ \\
\hline SJC_C097686 [CABF01092393] & + & 5.6 & $4249-4320$ \\
\hline
\end{tabular}

$(-)$ are contigs with 'signal sequence' on the negative strand (anti-sense) of the genome while

$(+)$ are contigs with 'signal sequence' on the positive strand (sense) in the genome

${ }^{*}$ Contigs are representative of disperse duplicated gene loci. We indicated the ranges for the signal sequence motif.

To investigate possible role by repetitive elements (REs) in mediating such dispersed duplication with a clue from previous studies [20,26-29], we performed repeat masking on the putative duplication source locus and the other 33 duplicons and observed a preponderance of flanking REs, especially of non-LTR class prominent of which were the S. japonicum RTE (retrotransposable element)-like retrotransposon (SjR2) and the Perere class of retrotransposons (SjR1) (Additional file 5). An almost full copy of SjR2 was found upstream of the coding region of the putative source locus in addition to other six albeit partial copies of SjR2. Alignment of the other contigs to the putative duplication source locus revealed that both the dispersed similar signal sequence and the repeat elements are considerably aligned at very similar positions, further showing that they were likely 
Table 4 Schistosoma japonicum Scaffolds containing the similar signal sequence $(n=18)$

\begin{tabular}{ll}
\hline SCaffolds [GenBank Accession] & Contigs within the sCaffolds \\
\hline SJC_S000013 [FN330988] & CABF01002611, CABF01002612, \\
& CABF01002622, CABF01002623, \\
& CABF01002628, CABF01002630 \\
SJC_S000219 [FN331192] & CABF01020047, CABF01020050 \\
SJC_S000220 [FN331193] & CABF01020060 \\
SJC_S000273 [FN331245] & CABF01022876, CABF01022884 \\
SJC_S000284 [FN331256] & CABF01023364 \\
SJC_S000329 [FN331301] & CABF01025296 \\
SJC_S000394 [FN331366] & CABF01027854, CABF01027861, \\
& CABF01027866 \\
SJC_S005820 [FN336777] & CABF01067176 \\
SJC_S007785 [FN338731] & CABF01070230 \\
SJC_S008639 [FN339578] & CABF01072590, CABF01072591 \\
SJC_S009177 [FN340103] & CABF01073691 \\
SJC_S010134 [FN341037] & CABF01075030 \\
SJC_S011206 [FN342077] & CABF01076032 \\
SJC_S011724 [FN342573] & CABF01078976 \\
SJC_S014521 [FN345237] & CABF01080674 \\
SJC_S014753 [FN345459] & CABF01080893 \\
SJC_S014868 [FN345568] & CABF01080757 \\
SJC_S026182 [FN354050] & CABF01092393
\end{tabular}

duplicated from a single source locus. The fact that the duplicons are not absolutely homologous and the degenerative nature of the RE sequences suggests variation within members, typical of evolving genes (Figure 4). Because homology with the other duplicates did not terminate 3' of this putative source locus, we recruited and adjoined two contigs [GenBank:CABF01020061 and GenBank:CABF01020062] downstream of the putative source locus according to the genome assembly information, thereby creating flanking sequences of at least 5 kilobasepairs on each side of the gene duplication source locus. This sequence was then aligned with the genome contigs and scaffolds to identify the exact point at which homology was lost, which could arguably represent the breakpoint of duplication. Further attempt to identify the exact breakpoints was not successful due to unfilled sequencing gaps in the scaffolds but examination of the downstream flanking sequence from the point where homology was terminated showed a prominent retrotransposon of the Perere class flanking the duplicated loci $3^{\prime}$ of the locus (see short movie in Additional file 5). Taken together, our data show that the duplication source locus was flanked on either side by RTElike and Perere class retrotransposons. These two classes of non-LTR retrotransposons have significantly high copy number, making up $12.63 \%$ of the $S$. japonicum
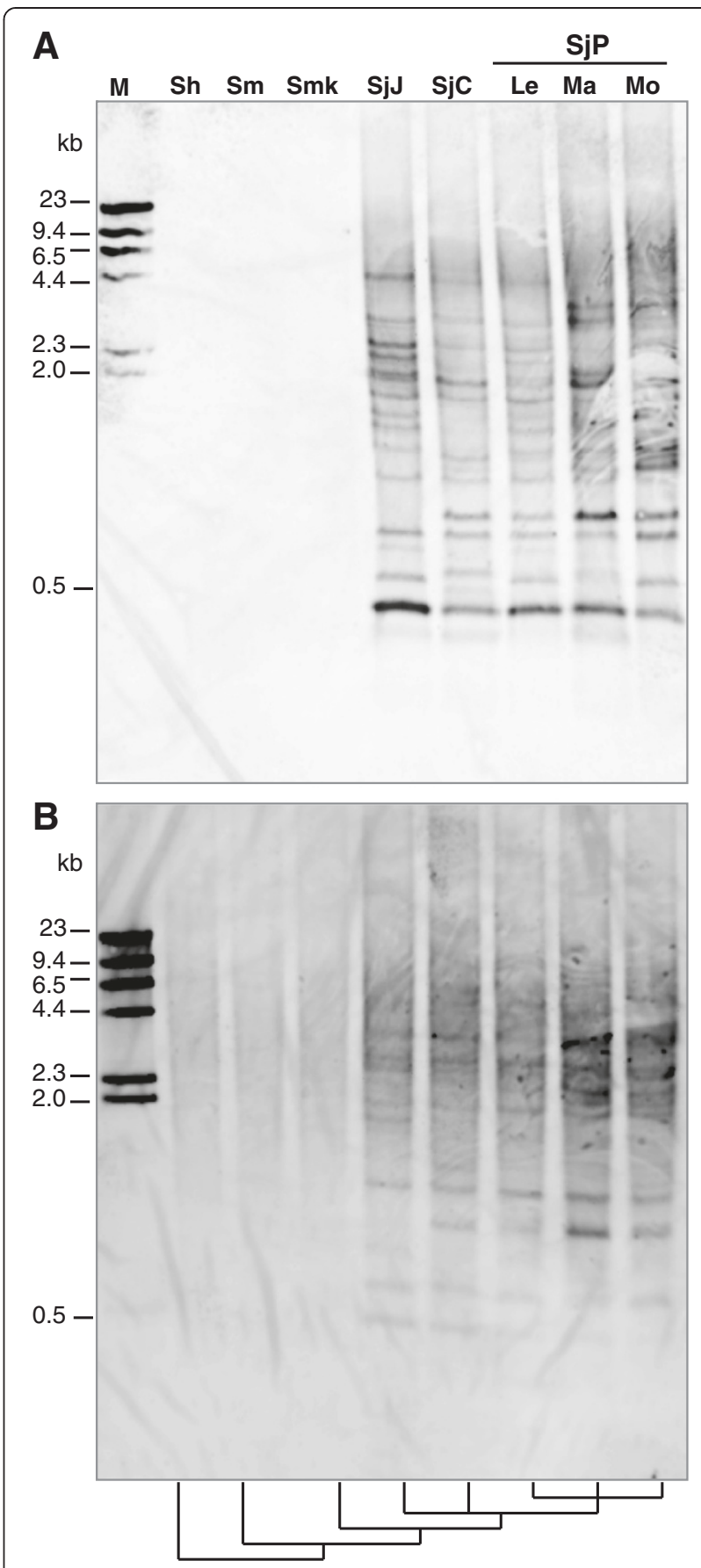

Figure 2 Southern blotting confirms duplicated loci exclusively in S. japonicum. (A) Southern hybridization with digoxigenin-labeled probes showing the presence of duplicated loci with several bands due to copies of the duplicated source locus. Lanes 2-9 corresponds to EcoRI + EcoRV double digested genomic DNA of different species and strains of Schistosoma (S. haematobium, S. mansoni, S. mekongi, S. japonicum (Japanese, Chinese, Philippines' Leyte, Mindanao and Mindoro isolates). ' $\mathrm{M}$ ' is Digoxigenin-labeled DNA molecular weight marker. Notice the differential banding pattern among different strains and between isolates of the same strain. (B) Same experiment as in (A) was replicated using a different pair of restriction enzymes (EcoRl + HindllI). Also interstrain and intra-strain variation in the banding pattern is apparent. 


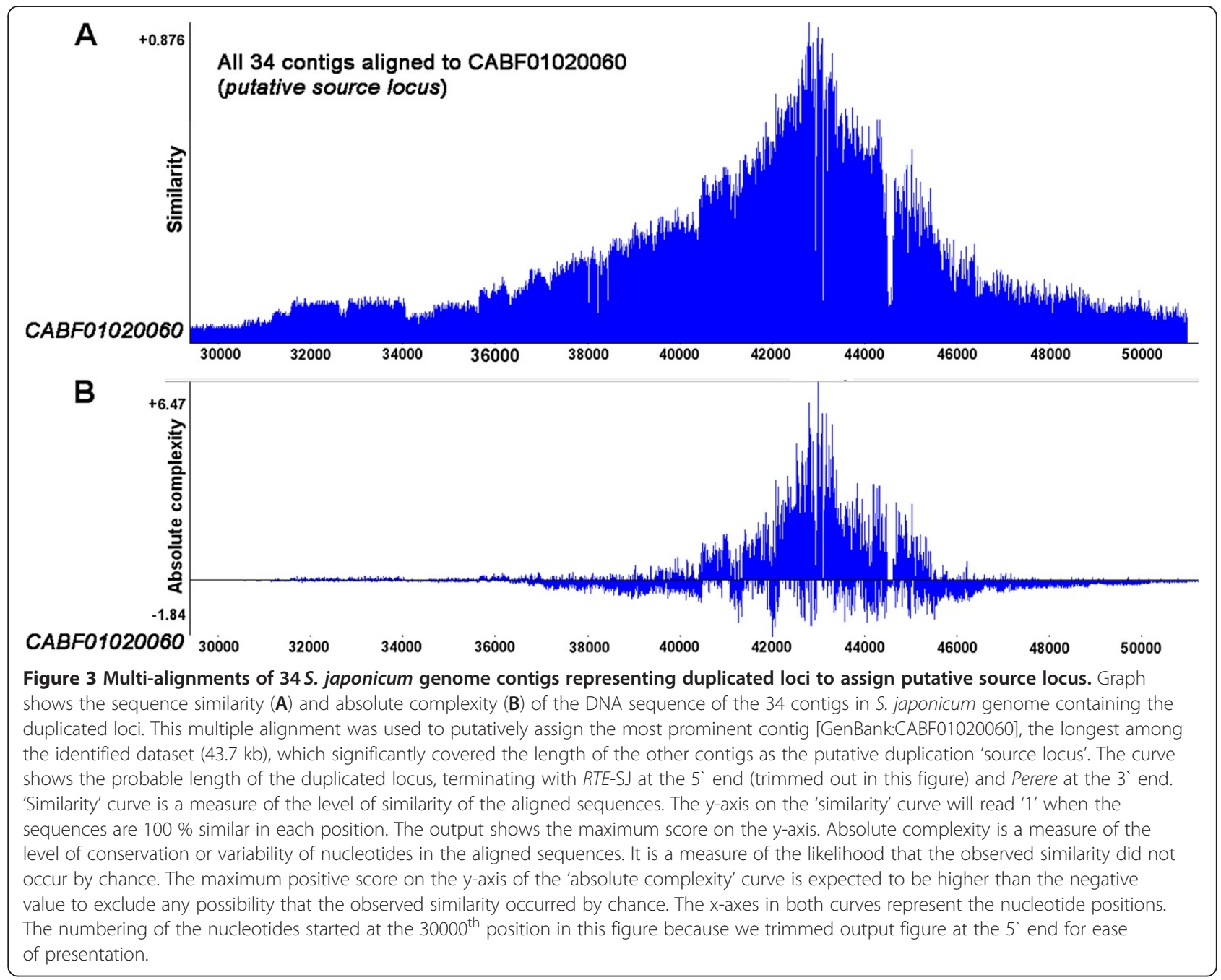

genome [49]. Different degrees of degeneracy of both the coding region and the flanking REs were observed in all the duplicated loci examined. This is consistent with the traditional view of the fate of new duplicons $[6,9]$, which assumes a tendency to be lost because of genetic drift under natural evolution [29,57] while not precluding the possibility for some duplicates to evolve distinct functions either by sub-functionalization or neo-functionalization.

The role of repetitive elements (REs) in dispersed duplication of genomic sequences is fairly documented from previous studies in model organisms [15,20,27,28,30,58,59]. The precise mechanism of this retrotransposon mediated dispersed duplication is not clear but may likely involve RE-mediated DNA level recombination, most likely by non-allelic homologous recombination (NAHR), alternatively called ectopic recombination (see illustration in Additional file 6). Due to their extremely high copy numbers, REs create structural modifications in the genome by providing the requisite highly similar DNA sequences, initiating recombination between non-allelic elements $[20,25,60]$, the result of which could be deletion, shuffling, duplication or transduction of a genomic DNA segment. Structural modifications introduced in the genome by NAHR mechanism can progress between non-homologous chromosomes (inter-chromosomal), between homologous chromosomes (inter-homologous or intra-chromosomal), between sister chromatids (inter-sister chromatid) or within a chromatid (intra-chromatid); giving rise to dispersed duplication of genomic segments, several forms of deletions or may create isodicentric chromosome by forming a mirrored segment in the chromosome by inversion. See detailed cartoon in Additional file 6. [60].

Many studies in other organisms have elucidated the role of REs in mediating sequence duplication, transduction and other structural variations by ectopic recombination mechanism. Notable among these is the human $A l u$ element for which several reports suggest a role in 


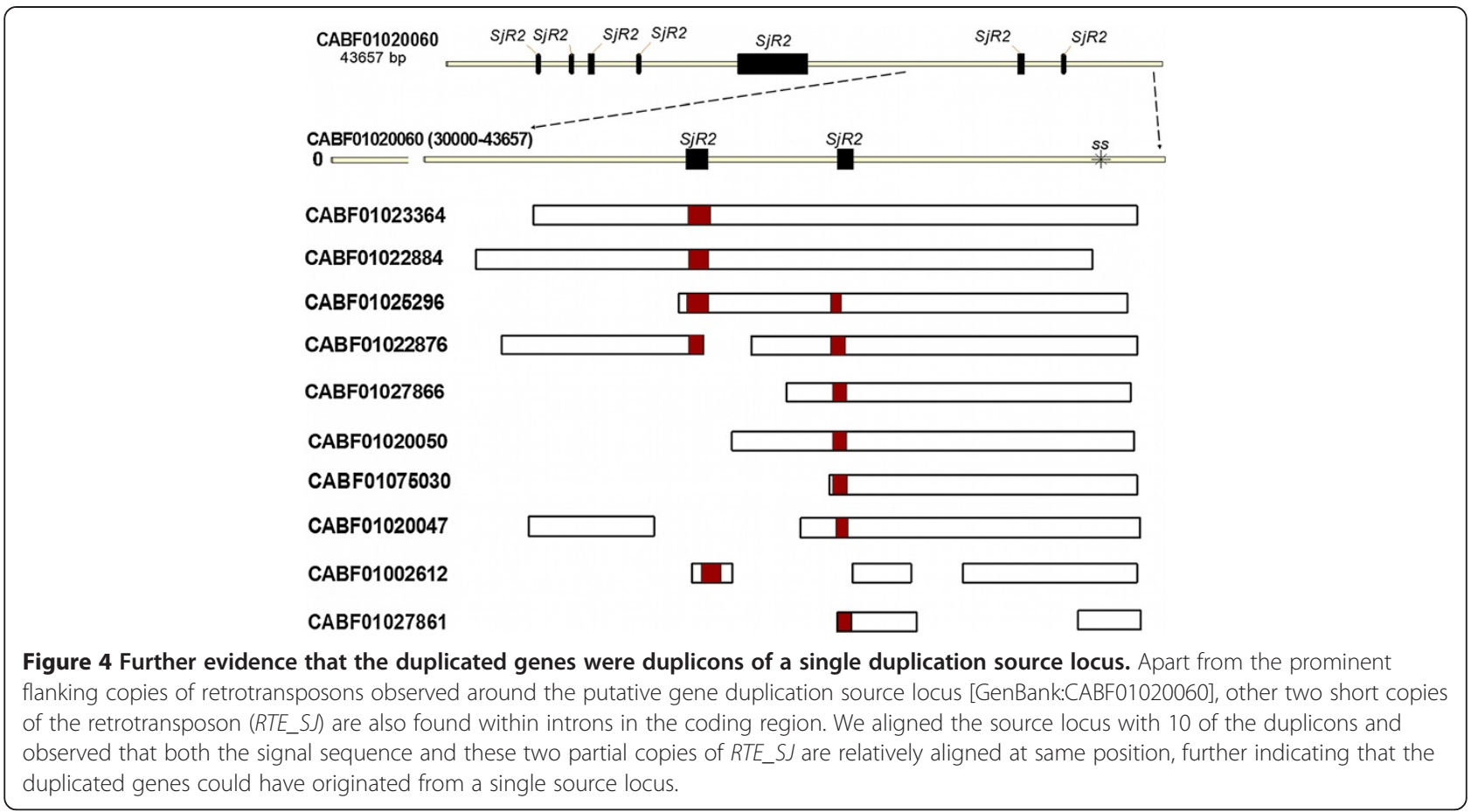

mediating NAHR and other structural modifications in the human genome [7,20,61]. Yang et al found an excess of repetitive sequences proximate to the breakpoints of duplicated gene loci in the genome of the fruit fly Drosophyla melanogaster, and have suggested that a NAHR mechanism, mediated by REs accounted for the birth of the new duplicons [1,27]. Another study performed on human individuals concluded that NAHR accounted for over $40 \%$ of detected genomic sequence duplications in the human genome [30]. Illegitimate recombination (IR), incomplete crossing over and nonhomologous end joining (NHEJ) are other possible mechanisms of gene duplication by DNA-level recombination, but NAHR play a more significant role in producing typical dispersed duplications [1] while the other mechanisms in addition to NAHR are more likely to produce tandem duplicates. Although we could not clearly identify the exact breakpoints of the duplications at both ends still for lack of a reference ancestral homolog and partly due to sequencing gaps, the fact that homology among all the scaffolds examined uniformly terminated at the same point with Perere on the $3^{`}$ end (Figure 3 and Additional file 5), and traces of the observed predominant retrotransposons (SjR2) was found at the exact positions as they occur in the putative source locus (Figure 4) confirm that these gene loci could be products of dispersed duplication from a single genomic source locus.

In addition to RE-mediated DNA-level recombination by NAHR, gene duplication events are also attributable to RE-mediated retrotransduction mechanism either on the $5{ }^{`}$ or $3{ }^{\prime}$ directions [27]. Xing et al and other groups have demonstrated the role of retrotransposons in the duplication of entire genes and creation of previously un-described genes by analyzing SVA (SINE, VNTR and Alu)-mediated retrotransduction events in the human genome $[20,29]$. However, we did not specifically identify any chimeric duplicon originating via a retrotransduction mechanism among our datasets. Furthermore, retrotransposons including SjR2 characteristically encode reverse transcriptase and endonuclease, and can therefore transcribe and 'paste' a gene sequence into new locations in the genome $[3,22,62]$. However, retrotransposed genes are characteristically intronless since the introns are usually spliced out during the process of retrotransposition. Our duplicons retained their introns, although in some case some portion of the introns may have either degenerated or deleted during duplication and subsequent sequence modifications $[3,22,63]$. A further evidence that a retrotransposition mechanism is unlikely in our observed cases was that while retrotransposons would not duplicate the promoter regions of duplicated gene based on the process of transcription and insertion of retrocopies [1,57] which leaves the newly retrotransposed sequences to acquire new regulatory sequences from adjacent genes or through mutations in order to be functional [14,19,24]; the protein coding duplicons observed among our duplicated gene loci retained the same or similar core regulatory region and signal sequence as the source locus, suggesting that they may not have been products of retroposition and 
may equally explain the parallel assumption of coding potential at their new duplication loci without the need to form chimeric structures with adjacent genes.

\section{Evolution of translatable ORF and evidence of expression of duplicated genes}

Some of the duplicons appear degenerative in homology and are relatively shorter than the source locus (Figure 3, also see Additional file 5) thus are consequently redundant and non-coding at the new locations as opined in the canonical view on the fate of new duplicons [6,9]; which assumes a tendency to be lost because of genetic drift under natural evolution $[29,57]$. However, our data provide evidence that some of the duplicons have evolved into protein coding genes with distinct products at their new loci, the fate of which could tend to either sub-functionalization to the source gene $[8,64]$ or neofunctionalization by acquiring new distinct functions $[9,65]$. In addition to the two duplicons with alternative splicing variants, which we further explored in the next section, some representatives of the protein coding duplicons were depicted in a supplementary figure (Additional file 7). The nucleotide sequences of these genes are still appreciably similar but accumulation of mutations and other sequence modifications have given rise to novel protein coding ORFs, encoding putatively distinct products. We identified and mapped each cDNA sequence to the genomic contigs using information we generated from GeneMark and GeneQuest gene predictions [66] and confirmed by alignment of the cDNAs to the genomic sequences using NCBI Spling program. This approach was necessitated because the fully mapped and annotated genome of S. japonicum is not presently available in the public databases. Intriguingly, our results corroborate the available UniGene and GenBank entries. Nevertheless, it is notable that we only assessed the duplicated copies on the basis of possessing the similar signal sequence. There is possibility that some other duplicons from this source locus could be involved in initiating other forms of structural modifications at other loci when incorporated into the coding region of other genes, but this was not investigated here.

To provide evidence of the transcription and expression of the putative source gene locus and some of the duplicons, we performed developmental stage specific RT-PCR using primers that specifically amplify the coding regions of the candidate genes from the cDNA libraries of each stage of $S$. japonicum. RT-PCR results provide evidence of the transcription of some of the duplicons at their new genomic sites in addition to the source locus (Figure 5). The candidate genes analyzed did not show differential developmental stage specific expression, although we did not perform quantitative estimation of expression levels. It is possible that this group represents a potential new family of proteins with similar signal peptides in this zoonotic trematode, which possess other extra distinctive characteristics from other members of the genus Schistosoma. We are presently undertaking further research to fully characterize the identified novel protein-coding genes to provide insight into the functional and structural significance of this trend in the genome of $S$. japonicum. The protein products of some of these candidate genes have already been expressed in our laboratory and confirmed by the reactivity of the immune sera with the parasite crude antigen preparations. The data will be reported with the molecular and functional characterization information.

\section{Functional selection by alternative splicing}

The precise recognition of exon-intron junction in a precursor mRNA (pre-mRNA) by the splicing machinery is central for the production of functional translatable mRNAs. However, there is often uncertainties in the choice of recognizable splice signals, resulting in a process termed alternative splicing [17], which enables the origination of multiple mRNA transcript variants from a single gene locus [67-69]. Alternative splicing mechanism could result in 'intronization' of an exon or 'exonization' of an intronic sequence. Ideally, the creation of an intron from a previously exonic sequence could lead to the loss of an ORF in coding genes. In evolving genes however, functional selection possibly by mutations may evolve the required splice signals and induce the intronization of an exon in a transcribed but non-coding mRNA gene sequence to create a translatable ORF encoding a functional protein. Conversely, while exonization of an intron could disrupt a translatable ORF in a coding gene, selective pressure may also evolve new splice signals within an intron to yield exons that could create a translatable ORF from a previously non-coding gene locus or a chimeric ORF from a protein-coding gene.

These two mechanisms have been shown from our observations to be capable of creating functional codinggenes from previously non-coding albeit transcribed mRNA sequences. We identified at least two classical evidences of alternative splicing and we propose that in addition to increasing coding potential and genomic diversity $[68,69]$, alternative splicing can also be one of the driving forces of adaptive evolution; producing genetic novelties and functional selection. The most prominent example of alternative splicing was observed in the duplication source locus [GenBank:CABF01020060], which was found to be able to produce a protein-coding mRNA [GenBank:AY570737] in addition to a noncoding mRNA transcript variant [GenBank:FN328299] (Figure 6). An alignment of the DNA sequences of these two transcripts with details of this observation is 


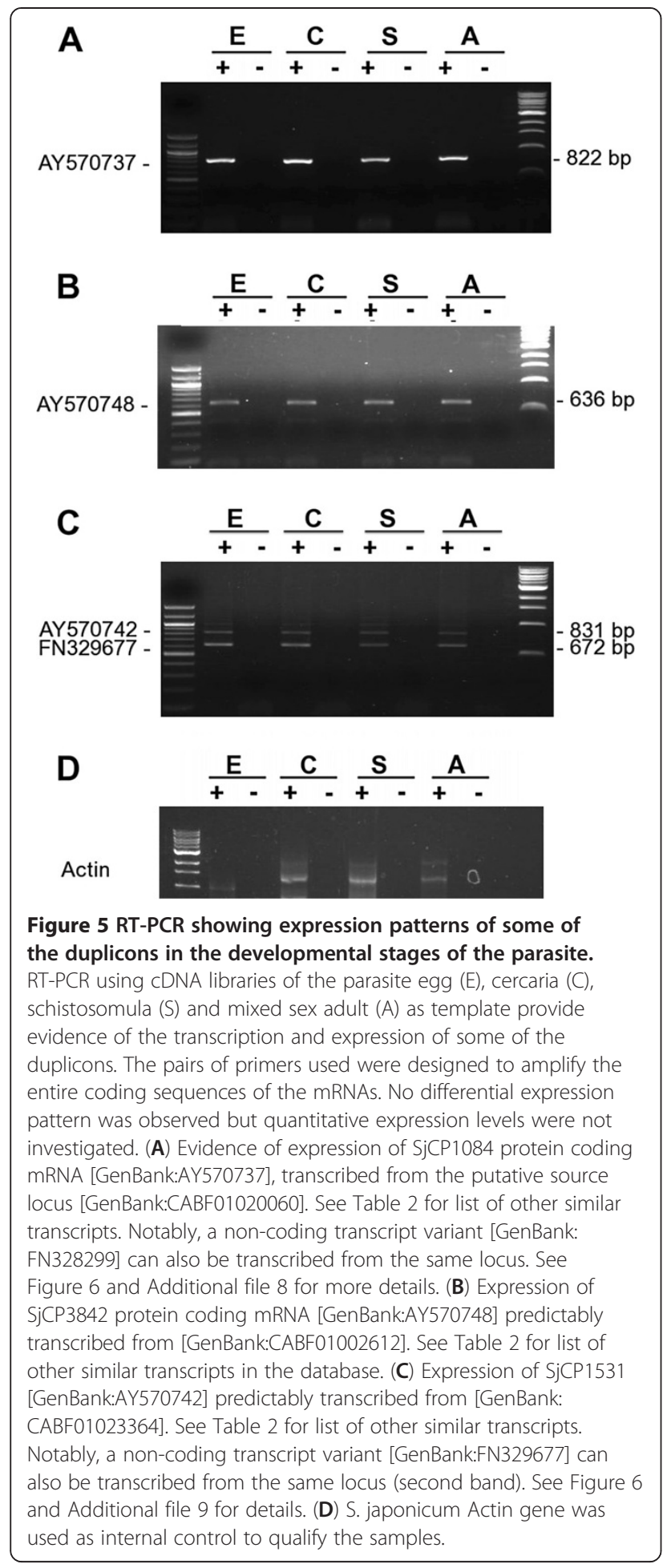

presented in the on-line published supporting information (Additional file 8). UniGene entries also suggest that the two transcripts are from the same locus (Table 2). An extra intron donor and acceptor sites were found within the first exon of the non-coding mRNA transcript
[GenBank:FN328299]. While the transcription model of the non-coding variant did not recognize the extra splice signals and thus retained the intron of about $1 \mathrm{~kb}$, the coding mRNA variant [GenBank:AY570737] recognized the splice sites and created an ORF from the gene by splicing out an intron thereby giving rise to the 5 'untranslated region (5 'UTR) and the first exon of a protein-coding gene encoding a protein product of 271 residues (SjCP1084). Additionally, another pair of splice acceptor and donor sites evolving at exon 5 of the noncoding variant resulted in the splicing out of a portion of the exon, all contributing in creating a translatable ORF in the protein coding variant (See Additional file 8 for details).

On the other hand, exons 5 and 6 of a coding mRNA variant [GenBank:AY570742] predictably transcribed from one of the progeny loci [GenBank:CABF01023364] were skipped in a non-coding shorter variant [GenBank: FN329677] without a translatable ORF (Figure 6 and Additional file 9). We observed that the sequences of exons 5 and 6 were similar and was repeated five times in tandem within this locus, but only two copies of the tandemly duplicated potential exons were incorporated into the coding sequence of the mRNA to create exons 5 and 6 of a protein-coding ORF of 274 codons (SjCP1531). These results represent typical models of alternative splicing by intronization and exonization respectively.

Although in evolutionary perspective, intron retention that creates a translatable ORF is considered more plausible than the reverse process; our data show that both mechanisms are potentially possible. Other groups have also identified intron gains recently in mammalian and rodent retrogenes $[68,69]$. The identification of noncoding mRNA variant alternatively transcribed from a single gene locus with a protein coding mRNA (Figure 6) is evidence that a novel protein-coding gene can originate from previously transcribed regions that contain the necessary transcription elements and provide RNA material for a protein translation machine $[2,39,68]$. Exon repetition has also been observed from our data to exist in this organism and could be instrumental in expanding the organism 's coding potential. The 'parallel' expression of the non-coding variant alongside the protein-coding transcripts is of significance and could suggest further that the gene may have been recently evolved. Noncoding RNAs have also been shown to perform some regulatory roles at various levels during gene expression $[2,68,70]$. This could be further explored with our data set. In the two described cases in our analyses, we have treated the non-coding isoforms as evolutionally preceding the coding variants; nevertheless, the reverse could also be the case. In addition to these two cases, we also identified a two-nucleotide insertion into a non-coding mRNA sequence [GenBank: FN330540] that yielded 


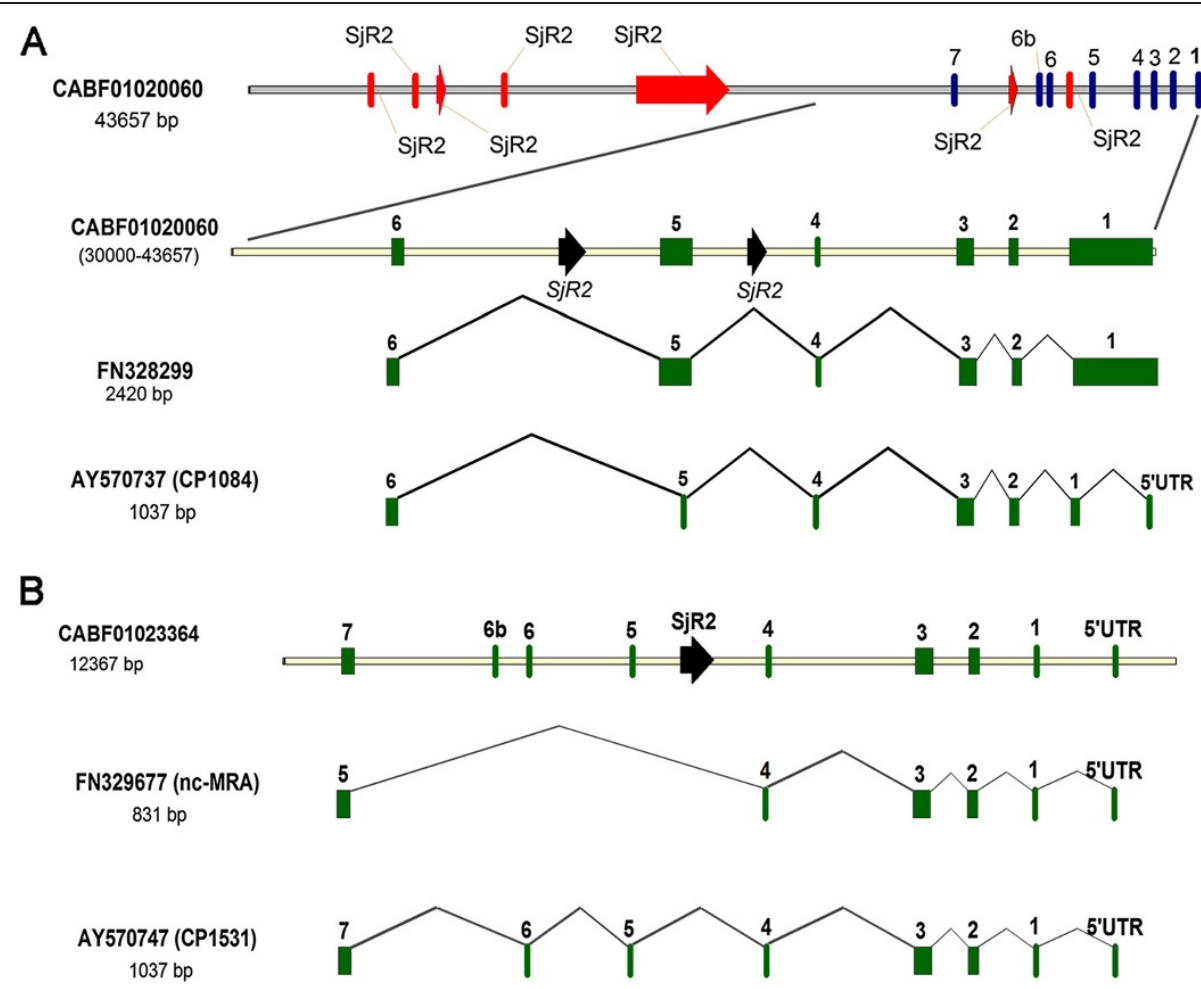

Figure 6 Splice models of some duplicons with evidence of alternatively splicing. (A) SjCP1084 protein coding mRNA [GenBank:AY570737] and a non-coding transcript [GenBank:FN328299] are products of alternative splicing. Based on gene prediction from the contigs using GeneQuest and GeneMark, and alignment of CDNAs to genome sequences using Spling program, we observed that two mRNA transcript variants were produced from [GenBank:CABF01020060]. An extra splice site was evolved in the first exon of the non-coding transcript [GenBank: FN328299]. When the splice site is recognized, an ORF encoding a protein coding mRNA [GenBank:AY570737] variant is created. The images were created from computer simulation of real DNA sequences using Vector NTI program. Also see a supplementary figure in Additional file 8 for more details. (B) SjCP1531 protein coding mRNA [GenBank:AY570742] and a non-coding transcript [GenBank:FN329677] are products of alternative splicing. Two mRNA transcript variants can be produced from a contig representing on of the duplicated loci [GenBank: CABF01023364]. Two extra splice sites were not utilized in the transcription of the non-coding transcript [GenBank:FN329677]. When the splice sites were recognized, exons 5 and 6 of a translatable ORF were created to produce a protein coding mRNA [GenBank:AY570742] variant. Refer to RT-PCR result in Figure 5 (C) where two bands of exact size and sequence as the two variants described above are apparent on the agarose gel electrophoresis image. Also see Additional file 9 for more details.

the coding mRNA of schistosomula protein with the similar signal peptide, with many similar transcripts in the database. However, this last observation could be an artifact from sequencing error since the existence of the non-coding transcript was not traceable to the genomic sequence.

\section{Conclusions}

We have passably delineated the possible mechanism that led to the identification of several protein coding genes with similar signal sequence, following lead from our work that isolated secreted proteins candidate genes using SST. A trend was described in the genome of S. japonicum whereby a 'newly evolved' gene served as a source locus for dispersed duplication events leading to the formation of several expressed genes with similar transcription core promoter region and signal sequence.
We further found that the duplicated gene locus was flanked by non-long terminal repetitive elements (REs), especially of the RTE-like and Perere class. We therefore inferred that REs may have played an important role in this dispersed gene duplication by creating the requisite homologous DNA sequence that mediate a DNA-level recombination, most probably by a non-allelic homologous recombination (NAHR) mechanism. Our findings also provide evidence of logical sequential process of novel gene origination by evolution of transcription core elements followed by translatable ORF. While similar RE mediated phenomena had been observed in other organisms, unlike our dataset, most analyses have centered on the model organisms. Our data contribute to the accumulating evidence that REs mediate diverse recombination events leading to novel gene origination and other evolutionary novelties. 


\section{Methods \\ BLAST search}

We had earlier identified a particular 81 nucleotides $(27$ amino acids) sequence, which was commonly utilized as signal sequence by several of our signal sequence trap (SST) isolated S. japonicum cDNAs (Table 1) [47]. The sequence of this signal sequence was employed as query to search for matches in the GenBank non-redundant nucleotide sequence database and expressed sequence tags (ESTs) database for all organisms using BLASTN program in National Center for Biotechnology Information (NCBI) Basic Local Alignment Search Tool (BLAST) [71]. A search on the NCBI UniGene database [72] that provides information on sets of transcript sequences that appear to come from the same transcription locus was performed to ascertain redundancy and group identified transcripts. For genome-wide searches, the same query sequence and program were used to search the WGS reads from all organisms with sequenced genomes deposited in the NCBI genome databases. In a similar search in the protein database, the amino acid and nucleotide sequence of the same signal sequence was used as query for BLASTP and BLASTX searches respectively. Conserved domain architecture searches on all translation products of the SST identified candidate genes were performed using the conserved domain architecture retrieval tool on NCBI website [73] and compared with same analyses on the ProDom database of protein domain families available online at [74].

\section{Multiple alignments}

All multiple sequence alignments of DNA and protein sequences were performed in parallel with ClustalW on MegAlign program in Lasergene 7 DNASTAR software, NCBI bl2seq, COBALT multiple alignment programs, and Multialin interface software [75]. cDNA-to-genome sequence alignments were computed using the free NCBI Spling program [75]. The latest update of the $S$. japonicum genome map is accessible at [52]. Phylogenetic and molecular evolutionary analyses were conducted using MEGA version 5 [76].

\section{Gene prediction}

Gene predictions were performed using the GeneQuest program (Lasergene 7 DNASTAR) to predict potential coding regions, starts, stops, acceptors and donor sites using Borodovsky matrix files for Caenorhabditis elegans; and the results compared with that of the Eukaryotic GeneMark.hmm [66] gene prediction server provided freely on the website of Georgia Institute of Technology, Atlanta, USA.

\section{Repeat masking}

The whole sequences of all the genome contigs bearing the similar signal sequence were screened against a reference collection of repetitive DNA elements in the RepBase database available at the Genomic Information Research Institute website, using the CENSOR repeat masking software [77]. Sequence analysis figures were generated using real DNA sequences on Vector NTI Advanced 11.0 (Invitrogen).

\section{Designation of putative duplication source locus and probable breakpoint}

Reference to a parent gene is required for accurate determination of duplication breakpoint. However, in absence of a reference homolog, we putatively selected the most prominent contig [GenBank:CABF01020060], the longest among the identified dataset $(43.7 \mathrm{~kb})$, which significantly covered the length of the other contigs (Figure 3, also see Additional file 5) as the putative duplication source locus and utilized it as such for most of the analyses performed in this study. When the contigs were aligned with the putative source locus, homology was not lost till the 3 ' end of the aligned sequences. We therefore recruited two contigs [GenBank:CABF01020061 and GenBank:CABF01020062] downstream of the source locus based on genome assembly information, thereby generating at least $5 \mathrm{~kb}$ flanking sequences on either side of the duplication source locus. This sequence was then aligned with the genome contigs and scaffolds to identify the exact point where sequence identity disappeared. This point was arguably chosen as the possible duplication breakpoint and utilized as such in our discussions. We further attempted to identify a recurrent consensus sequence at the breakpoints but this was hampered by several sequencing gaps in the partially assembled scaffolds.

\section{Parasites, genomic DNA and developmental stage mRNA samples}

Chinese strain of S. japonicum (hereafter abbreviated as $S j$ ) was obtained from Jiangsu Provincial Institute of Parasitic Diseases Wuxi, Jiangsu Province, PR China, while the Philippine and Japanese strains of S. japonicum in addition to $S$. mekongi (Smk) samples, were maintained in the Laboratory of Tropical Medicine and Parasitology, Dokkyo Medical University, Tochigi, Japan. S. mansoni $(\mathrm{Sm})$ adult worms were maintained by, and kindly provided by the Department of Parasitology, Institute of Tropical Medicine, Nagasaki University, Japan. S. haematobium (Sh) sample was from Department of Immunology and Parasitology, University of Occupational and Environmental Health, Kitakyushu, Japan. Total genomic DNA was purified from cut tissues of mixed sex adult worms from different species of 
Schistosoma using QIAamp DNA Mini Kit (QIAGEN) according to the manufacturer's instructions. Qualification and quantification of genomic DNA extract was assessed by gel electrophoresis and ND-1000 spectrophotometer (NanoDrop, USA). To obtain sufficient amount of genomic DNA for southern hybridization experiments, the whole genome of each sample was amplified using the GenomePhi DNA Amplification Kit (GE Healthcare) according to the manufacturer's instructions. Equally, total RNA was extracted from parasite eggs, cercariae, $24 \mathrm{~h}$ cultured schistosomulae and adult worms of $S$. japonicum according to the instruction manual of PureLink Micro-to-midi total RNA Purification System Kit (Invitrogen).

\section{Reverse transcription polymerase chain reaction (RT-PCR)}

mRNA from eggs, cercariae, 24 h culture schistosomulae and adult worms of the Chinese strain of S. japonicum was used for RT-PCR. The first strand cDNA was synthesized from the total RNA of each developmental stage by using oligo (dT) primer according to the instruction manual of High Capacity cDNA Reverse Transcription Kit (Applied Biosystems) and the resulting cDNA was used as template for RT-PCR. The S. japonicum actin gene was used for internal quality assurance. The cDNA sequences of some selected SST identified secreted candidate genes were amplified using pairs of sequence specific primers designed according to the S. japonicum transcriptome data [49] in the NCBI public database. All RT-PCR amplicons were analyzed using gel electrophoresis and confirmed by sequencing using the BigDye Terminator v1.1 Cycle Sequencing Kit (Applied Biosystem).

\section{Southern hybridization}

Southern hybridization was performed following standard procedures [78] using the DIG nonradioactive labeling and detection system (Roche, Germany). Briefly, the hybridization probe labeled with DIG-dUTP was synthesized using PCR DIG synthesis kit (Roche, Germany) according to the manufacturer's instructions, and labeling was confirmed by size disparity with unlabeled amplicon as a result of slower migration in agarose gel due to digoxigenin labeling. Genomic DNA from different species of Schistosoma (Sh, Sm, Smk, Sj Japanese (Yamanashi), Sj Chinese (Jiangsu) and $S j$ Philippines (Leyte, Mindanao and Mondoro isolates) were double digested with three different pairs of restriction enzymes $(E c o R I+E c o R V, \quad E c o R I+H i n d I I I$ and BamHI + HindIII $)$ to achieve the best possible fragmentation of the genomic DNA. The digested genomic DNA fragments were electrophoresed through $1 \%(w / v)$ agarose gel, depurinated in $250 \mathrm{mM} \mathrm{HCl}$, and denatured by incubating in two changes of denaturing solution for 15 min each $(0.5 \mathrm{M} \mathrm{NaOH}, 1.5 \mathrm{M} \mathrm{NaCl})$. The gels were then neutralized by incubation in two changes of neutralizing solution $(0.5 \mathrm{M}$ Tris- $\mathrm{HCl}$ at $\mathrm{pH} 7.5,1.5 \mathrm{M} \mathrm{NaCl})$ for $15 \mathrm{~min}$ each, and DNA was transferred to a positively charged nylon membrane (Roche, Germany) by capillary action overnight using 20x SSC solution $(3 \mathrm{M} \mathrm{NaCl}, 300 \mathrm{mM}$ sodium citrate at $\mathrm{pH}$ 7.0). The transferred DNA was fixed to the membrane by baking in an oven at $80^{\circ} \mathrm{C}$ for 2 hours after rinsing briefly in $2 x$ SSC. After prehybridizing the membrane in $10 \mathrm{ml}$ hybridization buffer (5x SSC, $0.1 \%$ N-lauroylsarcosine (w/v), $0.02 \% \operatorname{SDS}(\mathrm{w} / \mathrm{v}), 1 \%$ blocking solution (Roche, Germany)) for $30 \mathrm{mins}$ in a hybridization bag, $5 \mu \mathrm{l}$ of the PCR generated hybridization probe was mixed in $50 \mu \mathrm{l}$ of double deionized water, denatured by boiling for $5 \mathrm{mins}$ and introduced into the hybridization bag and incubated overnight with shaking at $50{ }^{\circ} \mathrm{C}$. The membrane was washed in two changes of low stringent wash buffer (2x SSC, $0.1 \%$ SDS) for 5 mins each at RT, and twice in high stringent wash buffers (0.5x SSC, $0.1 \%$ SDS) for 15 min each at $65{ }^{\circ} \mathrm{C}$. The hybridized probe was then detected using antiDigoxigenin antibody (Roche, Germany) using CSPD as the chemiluminiscent substrate according to the manufacturer's instructions. The blot was then visualized by exposing to chemiluminiscence for $10 \mathrm{~min}$ in a LAS-4000 mini image reader (Fujifilm).

\section{Additional files}

Additional file 1: Schematics of some of the mechanisms of novel
gene origination. Apart from the pioneering idea of gene duplication
[6], there are other mechanisms by which new genes are born. These
include but not limited to exon shuffling or exon "scrambling" (a) [4,14-
18]; fission or fusion of genes (b) [1,3,22], horizontal gene transfer
between organisms (c) [31-33], de novo origination of protein coding
genes from previously non-coding sequences (d) [2,3,34-40],
retrotransposition by retrotransposons yielding intronless chimeric genes
(e) [18-25], transduction of adjacent DNA by transposable elements (f) or
may involve a repetitive element mediated DNA level recombination by
a non-allelic homologous recombination (NAHR) mechanism (g) [7,20,26-
30]. The figure was adapted from [3].

Additional file 2: Multiple alignments of signal sequence trap (SST) isolated cDNAs showing similar signal sequence. The similar promoter region including the signal sequence is boxed. The two arrows indicate the 'ATG' start positions utilized in the transcript ORF of the candidate mRNA sequences.

Additional file 3: Phylogenetic tree of the genus Schistosoma showing the possible origination point of new duplicated genes. The species phylogeny was adapted from [53] as inferred from DNA sequencing, comparative molecular genomics and karyotyping. This phylogenetic tree was manually simulated and thus the length of the branches does not estimate dates or time scale. The tree shows the $S$. japonicum clade and a representative each of the other clades in the genus including the species that reinvaded Asia from Africa. See review in [53]. Based on the result of the southern hybridization in Figure 2, the species and strains that contain the duplicated genes encoding products with similar signal sequence are colored green and we inferred that the most probable time point estimate (black dot) of the gene's emergence could be after the other species in the S. japonicum group (in parenthesis) have diverged. 
Additional file 4: Expected fragments on restriction map of genome scaffolds correspond to bands on southern blots. To confirm dispersed duplication hypothesis and to exclude the possibilty of overlapping among the loci, the restriction map of six of the genome scaffolds bearing duplicated loci were generated (A). Using same restriction endonuclease enzymes as in the generated maps, we performed southern hybridization using restriction digested genomic DNA from S. japonicum species and strains, and were able to match the expected fragment sizes with the observed bands on the hybridization blots. The contigs and the expected probe binding sites were labeled followed by their sequence ranges. We denoted the respective restriction digested fragments with probe binding site using alphabets with their expected restriction digestion product sizes in parenthesis $(E+E$ : $E$ CoRI +EcoRV; $E+H: E c o R I+H$ ind III; $B+H=B a m H I+H i n d I I I)$. As shown in (B), we were able to match the expected fragment with the southern blot bands, labeled using their corresponding alphabetic codes. Probe binding site on the positive strand were colored 'green' while the antisense sites were colored 'red'. The tiny vertical lines on the graphics represent the cutting sites of the selected restriction enzymes. The restriction map and the image were generated using DNADynamo sequence analysis software.

Additional file 5: Simulations using our raw data to show DNALevel recombination mediated by REs by NAHR mechanism. The movie created from a Powerpoint presentation (Additional file 10) represents the basic approach we utilized in our analysis to show evidence of DNA level recombination by a non-allelic homologous recombination mechanism. The raw data obtained from BLAST searches and RepBase repetitive element prediction report was used to present simulation that demonstrates that the duplicated locus is flanked on $5^{\circ}$ and $3^{\prime}$ ends by retrotransposons of the classes RTE_SJ and Perere respectively. We proposed that these repetitive elements could have provided the requisite homologous stretch of DNA that is required for such DNA level recombination. NAHR can be inter-chromosomal, intrachromosomal, inter-sister chromatid, or intra-chromatid to give rise to disperse duplicates of the intervening genomic locus. This movie was created from an original Powerpoint presentation (Additional file 10)

\section{Additional file 6: A simplified illustration of repetitive element} mediated DNA level non-allelic homologous recombination (NAHR).

Repetitive elements provide the requisite homologous DNA sequence for DNA level recombination between non-allelic pairs by a NAHR mechanism. NAHR can occur within a chromosome (intra-homologous chromosomal), between chromosomes (inter-chromosomal), between sister-chromatids or within a chromatid to give rise to disperse duplicates of the intervening genomic locus. The figure was adapted from [60]. Also see Additional file 1 for a cartoon of NAHR and other mechanisms of new gene origination, and [20,26-29,60] for review.

\section{Additional file 7: Splicing models of some protein-coding} representatives of the young duplicons. Based on gene prediction from the contigs using GeneQuest and GeneMark and alignment of cDNAs to genome sequences using Spling program, we married the predicted products to the transcriptome database of this parasite and found that some of the duplicons are able to code for distinct gene products. Some of the transcription loci can encode two mRNA transcript variants. The significance of this was further explored in Figure 6.

Additional file 8: SjCP1084 protein coding mRNA [GenBank: AY570737] and a non-coding transcript [GenBank:FN328299] are products of alternative splicing Based on gene prediction from the contigs using GeneQuest and GeneMark, and alignment of cDNAs to genome sequences using Spling program, we observed that two mRNA transcript variants were produced from [GenBank:CABF01020060]. This figure is same as Figure 6 (A) but we have in addition presented the aligned sequence of the two transcripts showing details of alternative splicing. An extra splice site was evolved in the first exon of the noncoding transcript [GenBank:FN328299]. When the splice site is recognized, an ORF encoding SjCP1084 protein coding mRNA [GenBank:AY570737] variant is created.

Additional file 9: SjCP1531 protein coding mRNA [GenBank: AY570742] and a non-coding transcript [GenBank:FN329677] are products of alternative splicing. Based on gene prediction from the contigs using GeneQuest and GeneMark, and alignment of cDNAs to genome sequences using Spling program, we observed that two mRNA transcript variants were produced from [GenBank:CABF01023364]. This figure is same as Figure 6 (B) but we have in addition provided the aligned sequence of the two transcripts showing details of alternative splicing. Two extra splice sites were not utilized in the transcription of the non-coding transcript [GenBank:FN329677]. When the splice sites were recognized, exons 5 and 6 of a translatable ORF were created to produce SjCP1531 protein coding mRNA [GenBank:AY570742] variant. Refer to RT-PCR result in Figure 5 (C) where two bands (exact size and sequence as the two variants described above) are seen on the agarose gel electrophoresis image.

Additional file 10: Simulations using our raw data to show DNALevel recombination mediated by REs by NAHR mechanism. This Powerpoint presentation was used to create the movie in Additional file 5 .

\section{Abbreviations}

SST: Signal sequence trap; NAHR: Non-allelic homologous recombination; DLR: DNA level recombination; NHEJ: Non-homologous end joining; IR: Illegitimate recombination; RE: Repetitive element; ORF: Open reading frame; WGS: Whole genome shotgun.

\section{Competing interests}

The authors declare that they have no competing interests.

\section{Author contributions}

ECM participated in the conception and design of the study, in-silico analyses, molecular experiments, data analysis and interpretation and drafted the manuscript. YC carried out the signal sequence trap (SST) and participated in in-silico analyses. MK participated in the design of the study, SST, in-silico analyses, molecular experiments and data interpretation. MNS participated in in-silico analyses, molecular experiments, data interpretation and revised the manuscript. DB participated in molecular experiments and data analyses. $\mathrm{MK}_{2}, \mathrm{NH}_{1} \mathrm{YC}_{2}$ and $\mathrm{YO}$ maintained parasite life cycle and participated in molecular experiments. SH participated in data interpretation, supervision and revised manuscript for intellectual content. KH participated in the conception and design of the study, SST, in-silico analyses, data interpretation, revised the manuscript and general coordination. All authors approved final version of the manuscript.

\section{Acknowledgements}

We would like to thank Prof. Kenji Hirayama's lab members (Department of Immunogenetics, Institute of Tropical Medicine, Nagasaki University) for insightful discussions. ECM is a recipient of the Japanese Government Ministry of Education, Culture, Sports, Science and Technology (MEXT) PhD fellowship. This study was supported in part by the Global Center of Excellence (GCOE) Program (2008-2011); Grant-in-Aid for $21^{\text {st }}$ century COE program (2003-2008), Nagasaki University; and Grant-in-Aid for Scientific Research B (22406009) and C (23590489) from the Japanese Government Ministry of Education, Culture, Sports, Science and Technology (MEXT). The funding agency played no role in conducting the study, drafting the manuscript and the decision to publish.

\section{Author details}

'Department of Immunogenetics, Institute of Tropical Medicine (NEKKEN), and Global COE Program, Nagasaki University, 1-12-4 Sakamoto, 852-8523, Nagasaki, Japan. ${ }^{2}$ Laboratory on Technology for Parasitic Disease Prevention and Control, Jiangsu Institute of Parasitic Diseases, 117 Yangxiang, Meiyuan, Wuxi 214064, People's Republic of China. 'Laboratory of Tropical Medicine and Parasitology, Dokkyo Medical University, Tochigi, Japan. ${ }^{4}$ Department of Immunology and Parasitology, The University of Occupational and Environmental Health, Kitakyushu, Japan. ${ }^{5}$ Department of Parasitology, Institute of Tropical Medicine (NEKKEN), and Global COE Program, Nagasaki University, 1-12-4 Sakamoto, 852-8523, Nagasaki, Japan. ${ }^{6}$ Department of Parasitology and Entomology, Faculty of Bioscience, Nnamdi Azikiwe University, P.M.B. 5025, Awka, Nigeria.

Received: 2 February 2012 Accepted: 11 June 2012

Published: 20 June 2012 


\section{References}

1. Zhou Q, Wang W: On the origin and evolution of new genes-a genomic and experimental perspective. Journal of Genetics and Genomics 2008, 35(11):639-648.

2. Cai J, Zhao R, Jiang H, Wang W: De novo origination of a new proteincoding gene in Saccharomyces cerevisiae. Genetics 2008, 179(1):487-496.

3. Long $\mathrm{M}$, Betran $\mathrm{E}$, Thornton $\mathrm{K}$, Wang $\mathrm{W}$ : The origin of new genes: glimpses from the young and old. Nat Rev Genet 2003, 4(11):865-875.

4. Long M, Deutsch $M$, Wang W, Betrán E, Brunet FG, Zhang J: Origin of New Genes: Evidence from Experimental and Computational Analyses. Genetica 2003, 118(2):171-182.

5. Kaessmann H: Origins, evolution, and phenotypic impact of new genes. Genome Res 2010, 20(10):1313-1326.

6. Ohno S: Evolution by gene duplication. New York: Springer-Verlag; 1970.

7. Bailey JA, Liu G, Eichler EE: An Alu transposition model for the origin and expansion of human segmental duplications. Am J Hum Genet 2003, 73(4):823-834.

8. Gao X, Lynch M: Ubiquitous internal gene duplication and intron creation in eukaryotes. Proc Natl Acad Sci 2009, 106(49):20818-20823.

9. Katju V, Lynch M: On the formation of novel genes by duplication in the Caenorhabditis elegans genome. Mol Biol Evol 2006, 23(5):1056-1067.

10. Gu Z, Rifkin SA, White KP, Li W-H: Duplicate genes increase gene expression diversity within and between species. Nat Genet 2004, 36(6):577-579

11. Innan $H$, Kondrashov F: The evolution of gene duplications: classifying and distinguishing between models. Nat Rev Genet 2010, 11(2):97-108.

12. Osada N, Innan H: Duplication and gene conversion in the Drosophila melanogaster genome. PLoS genetics 2008, 4(12):e1000305

13. Zhou Q, Zhang G, Zhang Y, Xu S, Zhao R, Zhan Z, Li X, Ding Y, Yang S, Wang W: On the origin of new genes in Drosophila. Genome Res 2008, 18(9):1446-1455.

14. Long $\mathrm{M}$, Langley $\mathrm{CH}$ : Natural selection and the origin of jingwei, a chimeric processed functional gene in Drosophila. Science 1993, 260(5104):91-95.

15. Moran JV, DeBerardinis RJ, Kazazian HH Jr: Exon shuffling by L1 retrotransposition. Science 1999, 283(5407):1530-1534.

16. Patthy L: Genome evolution and the evolution of exon-shuffling-a review. Gene 1999, 238(1):103-114.

17. Shao $X$, Shepelev V, Fedorov A: Bioinformatic analysis of exon repetition, exon scrambling and trans-splicing in humans. Bioinformatics 2006, 22(6):692-698.

18. Elrouby N, Bureau TE: Bs1, a New Chimeric Gene Formed by Retrotransposon-Mediated Exon Shuffling in Maize. Plant Physiol 2010, 153(3):1413-1424

19. Wang $W$, Brunet $F G$, Nevo E, Long M: Origin of sphinx, a young chimeric RNA gene in Drosophila melanogaster. Proc Natl Acad Sci U S A 2002, 99(7):4448-4453.

20. Cordaux R, Batzer MA: The impact of retrotransposons on human genome evolution. Nat Rev Genet 2009, 10(10):691-703.

21. Courseaux A, Nahon JL: Birth of two chimeric genes in the Hominidae lineage. Science 2001, 291(5507):1293-1297.

22. Kaessmann $\mathrm{H}$, Vinckenbosch $\mathrm{N}$, Long M: RNA-based gene duplication: mechanistic and evolutionary insights. Nat Rev Genet 2009, 10(1):19-31.

23. Shapiro J: Transposable elements as the key to a 21st century view of evolution. Genetica 1999, 107(1):171-179.

24. Okamura K, Nakai K: Retrotransposition as a Source of New Promoters. Mol Biol Evol 2008, 25(6):1231-1238.

25. Hancks DC, Goodier JL, Mandal PK, Cheung LE, Kazazian HH Jr: Retrotransposition of marked SVA elements by human L1s in cultured cells. Hum Mol Genet 2011, 20(17):3386-3400.

26. Johnson ME, Cheng Z, Morrison VA, Scherer S, Ventura M, Gibbs RA, Green $E D$, Eichler EE: Recurrent duplication-driven transposition of DNA during hominoid evolution. Proc Natl Acad Sci U S A 2006, 103(47):17626-17631.

27. Yang S, Arguello JR, Li X, Ding Y, Zhou Q, Chen Y, Zhang Y, Zhao R, Brunet F, Peng $L$, et al: Repetitive Element-Mediated Recombination as a Mechanism for New Gene Origination in Drosophila. PLoS genetics 2008, 4(1):e3.

28. Shapiro JA: A 21st century view of evolution: genome system architecture, repetitive DNA, and natural genetic engineering. Gene 2005, 345(1):91-100.

29. Xing J, Wang H, Belancio VP, Cordaux R, Deininger PL, Batzer MA: Emergence of primate genes by retrotransposon-mediated sequence transduction. Proc Natl Acad Sci 2006, 103(47):17608-17613.

30. Kidd JM, Cooper GM, Donahue WF, Hayden HS, Sampas N, Graves T, Hansen N, Teague B, Alkan C, Antonacci F, et al: Mapping and sequencing of structural variation from eight human genomes. Nature 2008 453(7191):56-64
31. Eugene V, Koonin KSM, Aravind L: Horizontal Gene Transfer in Prokaryotes: Quantification and Classification. Annu Rev Microbiol 2001, 55:709-742.

32. Hotopp JCD, Clark ME, Oliveira DCSG, Foster JM, Fischer P, Torres MCM, Giebel JD, Kumar N, Ishmael N, Wang S, et al: Widespread Lateral Gene Transfer from Intracellular Bacteria to Multicellular Eukaryotes. Science 2007, 317(5845):1753-1756.

33. Keeling PJ, Palmer JD: Horizontal gene transfer in eukaryotic evolution. Nat Rev Genet 2008, 9(8):605-618.

34. Yang Z, Huang J: De novo origin of new genes with introns in Plasmodium vivax. FEBS Lett 2011, 585(4):641-644.

35. Levine MT, Jones CD, Kern AD, Lindfors HA, Begun DJ: Novel genes derived from noncoding DNA in Drosophila melanogaster are frequently X-linked and exhibit testis-biased expression. Proc Natl Acad Sci 2006, 103(26):9935-9939.

36. Volff J-N: Turning junk into gold: domestication of transposable elements and the creation of new genes in eukaryotes. BioEssays 2006, 28(9):913-922.

37. Begun DJ, Lindfors HA, Kern AD, Jones CD: Evidence for de Novo Evolution of Testis-Expressed Genes in the Drosophila yakuba/ Drosophila erecta Clade. Genetics 2007, 176(2):1131-1137.

38. Knowles DG, McLysaght A: Recent de novo origin of human proteincoding genes. Genome Res 2009, 19(10):1752-1759.

39. Wu DD, Irwin DM, Zhang YP: De novo origin of human protein-coding genes. PLoS genetics 2011, 7(11):e1002379.

40. Guerzoni D, McLysaght A: De novo origins of human genes. PLoS genetics 2011, 7(11):e1002381.

41. Snel B, Bork P, Huynen M: Genome evolution. Gene fusion versus gene fission. Trends Genet 2000, 16(1):9-11.

42. Wilson MS, Mentink-Kane MM, Pesce JT, Ramalingam TR, Thompson R, Wynn TA: Immunopathology of schistosomiasis. Immunol Cell Biol 2006, 85(2):148-154

43. Cass CL, Johnson JR, Califf LL, X X T, Hernandez HJ, Stadecker MJ, Yates JR III, Williams DL: Proteomic analysis of Schistosoma mansoni egg secretions. Mol Biochem Parasitol 2007, 155(2):84-93.

44. Edungbola LD, Cha YN, Bueding E, Schiller EL: Granuloma formation around exogenous eggs of Schistosoma mansoni and Schistosoma japonicum in mice. Afr J Med Med Sci 1982, 11(2):75-79.

45. Burke ML, Jones MK, Gobert GN, Li YS, Ellis MK, McManus DP: Immunopathogenesis of human schistosomiasis. Parasite Immunology 2009, 31(4):163-176.

46. McManus DP, Loukas A: Current Status of Vaccines for Schistosomiasis. Clin Microbiol Rev 2008, 21(1):225-242.

47. Yu C, Zhang F, Yin X, Kikuchi M, Hirayama K: Isolation of the cDNAs encoding secreted and membrane binding proteins from egg of Schistosoma japonicum (Chinese strain). Acta Parasitologica 2008, 53(1):110-114.

48. Spanu PD, Abbott JC, Amselem J, Burgis TA, Soanes DM, Stüber K, Loren van Themaat EV, Brown JKM, Butcher SA, Gurr SJ, et al: Genome Expansion and Gene Loss in Powdery Mildew Fungi Reveal Tradeoffs in Extreme Parasitism. Science 2010, 330(6010):1543-1546.

49. Feng Liu YZ, Wang Zhi-Qin, Lu Gang, Zheng Huajun, Brindley Paul J, McManus Donald P, Blair David, Zhang Qin-hua, Zhong Yang, Wang Shengyue, Han Ze-Guang, Chen Zhu: The Schistosoma japonicum genome reveals features of host-parasite interplay. Nature 2009, 460:345-351 (16 July 2009).

50. Hu W, Brindley PJ, McManus DP, Feng Z, Han Z-G: Schistosome transcriptomes: new insights into the parasite and schistosomiasis. Trends in Molecular Medicine 2004, 10(5):217-225.

51. Hu W, Yan Q, Shen DK, Liu F, Zhu ZD, Song HD, Xu XR, Wang ZJ, Rong YP, Zeng $L C$, et al: Evolutionary and biomedical implications of a Schistosoma japonicum complementary DNA resource. Nat Genet 2003, 35(2):139-147

52. Gene DB; [http://www.genedb.org/Homepage/Sjaponicum].

53. Lawton SP, Hirai H, Ironside JE, Johnston DA, Rollinson D: Genomes and geography: genomic insights into the evolution and phylogeography of the genus Schistosoma. Parasit Vectors 2011, 4:131.

54. Lockyer AE, Olson PD, Ostergaard P, Rollinson D, Johnston DA, Attwood SW, Southgate VR, Horak P, Snyder SD, Le TH, et al: The phylogeny of the Schistosomatidae based on three genes with emphasis on the interrelationships of Schistosoma Weinland, 1858. Parasitology 2003, 126(Pt 3):203-224. 
55. Le TH, Humair PF, Blair D, Agatsuma T, Littlewood DT, McManus DP: Mitochondrial gene content, arrangement and composition compared in African and Asian schistosomes. Mol Biochem Parasitol 2001, 117(1):61-71.

56. Chen ST, Cheng HC, Barbash DA, Yang HP: Evolution of hydra, a recently evolved testis-expressed gene with nine alternative first exons in Drosophila melanogaster. PLoS genetics 2007, 3(7):e107.

57. Szak ST, Pickeral OK, Landsman D, Boeke JD: Identifying related L1 retrotransposons by analyzing 3' transduced sequences. Genome Biol 2003, 4(5):R30

58. Wang W, Zheng H, Fan C, Li J, Shi J, Cai Z, Zhang G, Liu D, Zhang J, Vang S, et al: High Rate of Chimeric Gene Origination by Retroposition in Plant Genomes. The Plant Cell Online 2006, 18(8):1791-1802.

59. Thomas JH, Schneider S: Coevolution of retroelements and tandem zinc finger genes. Genome Res 2011, 21(11):1800-1812.

60. Sasaki M, Lange J, Keeney S: Genome destabilization by homologous recombination in the germ line. Nat Rev Mol Cell Biol 2010, 11(3):182-195.

61. Cordaux R: The human genome in the LINE of fire. Proc Natl Acad SC U S A 2008, 105(49):19033-19034.

62. Laha T, Brindley PJ, Smout MJ, Verity CK, McManus DP, Loukas A: Reverse transcriptase activity and untranslated region sharing of a new RTE-like, non-long terminal repeat retrotransposon from the human blood fluke, Schistosoma japonicum. Int J Parasitol 2002, 32(9):1163-1174

63. Zhang YE, Vibranovski MD, Krinsky BH, Long M: A cautionary note for retrocopy identification: DNA-based duplication of intron-containing genes significantly contributes to the origination of single exon genes. Bioinformatics 2011, 27(13):1749-1753.

64. Lynch M, Force A: The probability of duplicate gene preservation by subfunctionalization. Genetics 2000, 154(1):459-473.

65. Conant GC, Wolfe KH: Turning a hobby into a job: How duplicated genes find new functions. Nat Rev Genet 2008, 9(12):938-950.

66. Lomsadze A, Ter-Hovhannisyan V, Chernoff YO, Borodovsky M: Gene identification in novel eukaryotic genomes by self-training algorithm. Nucleic Acids Res 2005, 33(20):6494-6506.

67. Modrek B, Lee C: A genomic view of alternative splicing. Nat Genet 2002, 30(1):13-19.

68. Keren H, Lev-Maor G, Ast G: Alternative splicing and evolution: diversification, exon definition and function. Nat Rev Genet 2010 11(5):345-355

69. Szczesniak MW, Ciomborowska J, Nowak W, Rogozin IB, Makalowska I: Primate and rodent specific intron gains and the origin of retrogenes with splice variants. Mol Biol Evol 2011, 28(1):33-37.

70. Mattick JS, Makunin IV: Non-coding RNA. Hum Mol Genet 2006, 15(Spec No 1):R17-R29.

71. NCBI: Basic Local Alignment Search Tool (BLAST): [http://blast.ncbi.nlm.nih. gov/Blast.cgi].

72. NCBI: UniGene Database; [http://www.ncbi.n/m.nih.gov/unigene? term=txid6182[organism].

73. NCBI: Conserved Domain Architecture Retrieval Tool: [http://www.ncbi.nlm.nih. gov/Structure/cdd/cdd.shtml].

74. ProDom Database: [http://prodom.prabi.fr/prodom/current/html/home.php].

75. Corpet F: Multiple sequence alignment with hierarchical clustering. Nucleic Acids Res 1988, 16(22):10881-10890.

76. Tamura K, Peterson D, Peterson N, Stecher G, Nei M, Kumar S: MEGA5: Molecular Evolutionary Genetics Analysis Using Maximum Likelihood, Evolutionary Distance, and Maximum Parsimony Methods. Mol Biol Evol 2011, 28(10):2731-2739.

77. Kohany O, Gentles AJ, Hankus L, Jurka J: Annotation, submission and screening of repetitive elements in Repbase: RepbaseSubmitter and Censor. BMC Bioinforma 2006, 7:474

78. Southern E: Southern blotting. Nat Protoc 2006, 1(2):518-525.

doi:10.1186/1471-2164-13-260

Cite this article as: Mbanefo et al:: Origin of a novel protein-coding gene family with similar signal sequence in Schistosoma japonicum. BMC Genomics 2012 13:260.

\section{Submit your next manuscript to BioMed Central and take full advantage of:}

- Convenient online submission

- Thorough peer review

- No space constraints or color figure charges

- Immediate publication on acceptance

- Inclusion in PubMed, CAS, Scopus and Google Scholar

- Research which is freely available for redistribution

Submit your manuscript at www.biomedcentral.com/submit
C Biomed Central 